\title{
Analysis of Airflow Imbalances in an Open Compute High Density Storage Data Center
}

\author{
${ }^{1}$ Husam A. Alissa*, ${ }^{1}$ Kourosh Nemati, ${ }^{2}$ Udaya L.N. Puvvadi, ${ }^{1}$ Bahgat G. Sammakia Fellow, IEEE, ${ }^{3}$ Ken Schneebeli, \\ ${ }^{4}$ Mark Seymour, ${ }^{4}$ Tom Gregory \\ ${ }^{1}$ Department of Mechanical Engineering, ES2 Center, Binghamton University-SUNY, NY, USA \\ ${ }^{2}$ Department of Computer Science, ES2 Center, Binghamton University-SUNY, NY, USA \\ ${ }^{3}$ IBM Corporation, San Jose, CA, USA \\ ${ }^{4}$ Future Facilities Ltd, London, UK \\ E-mail*: halissa1@,binghamton.edu
}

Abstract

This article reports experimental and numerical testing performed to characterize the operation and reliability of the open compute (OC) storage system in contained environment from server to aisle levels. The study is comprised of three parts. The first part is an experimental analysis of the high density (HD) 3D array storage unit thermal and utilization responses during airflow imbalances. This is done with the stress test proposed for IT in containment to mimic possible mismatch and cascade failure scenarios. It is found that downstream HDDs are most prone to overheating and loss in utilization during an airflow imbalance. This was proven to undermine the storage capacity of the hard disk drives. An IT level airflow prediction model is discussed for the storage unit and validated for different fan speeds. In the second part, a computational fluid dynamics model is created for a high density open rack based on the active flow curve method. Here, the measured airflow response curves for the open compute IT (storage and compute servers) are used to build compact models and run rack level testing for IT air systems sensitivity and create a rack level AFC (active flow curve) airflow demand prediction model. Finally, the experimental characterization data is used to build an aisle level model (POD) that incorporates IT fan control systems (FCS). This modeling approach yields shorter uptime during chiller failure due to increased recirculation induced by increased IT airflow demand during cases such as chiller failure or high economizer temperatures.

Keywords: Storage, High Density, IT, Containment, HDD, Open Compute, Reliability, flow curves, Utilization.

\author{
Nomenclature \\ A Proportional Multiplier or Area \\ AF Acceleration Factor \\ AFC Active Flow Curve \\ ARFS Average Rack Fans' Speed \\ ASIC Application-Specific Integrated Circuit \\ CAC Cold Aisle Containment \\ $C_{p} \quad$ Specific heat at constant pressure \\ CPU Central Processing Unit \\ $\mathrm{D}_{\mathrm{h}} \quad$ Hydraulic diameter [m] \\ DPB Drive Plane Board \\ DRAM Dynamic Access Random Memory \\ $\mathrm{Ea} \quad$ Activation Energy [eV] \\ EB Exabyte (1 billion Gigabytes) \\ FCB Fan Control Board \\ FCS Fan Control System \\ FD Free Delivery [CFM] \\ $F_{i} \quad$ Body force in Cartesian direction $i$ \\ HAC Hot Aisle Containment \\ HBA Hot Bus Adapter \\ HD High Density \\ HDD Hard Disk Drive \\ IPMI Intelligent Platform Management Interface \\ IT Information Technology, (also refers to servers, storages, and switches)
}




\begin{tabular}{|c|c|}
\hline $\mathrm{K}$ & Boltzmann's constant $\left[\mathrm{eV} /{ }^{\circ} \mathrm{K}\right]$ \\
\hline K & Effective thermal conductivity \\
\hline JBOD & Just a Bunch Of Disks \\
\hline $\mathrm{K}_{\mathrm{i}, \mathrm{v}}$ & Modified inertial $\left[\right.$ in. $\left.\mathrm{H}_{2} \mathrm{O} / \mathrm{CFM}^{2}\right]$ and viscous $\left[\right.$ in. $\mathrm{H}_{2} \mathrm{O} / \mathrm{CFM}$ ] loss coefficients \\
\hline $\mathrm{K}_{1,2}$ & Inertial [in. $\mathrm{H}_{2} \mathrm{O} / \mathrm{CFM}^{2}$ ] and viscous [in. $\mathrm{H}_{2} \mathrm{O} / \mathrm{CFM}$ ] loss coefficients \\
\hline$\mu$ & Dynamic viscosity $[\mathrm{kg} / \mathrm{m} . \mathrm{s}]$ \\
\hline $\mathrm{N}$ & Fan speed \\
\hline NFS & Network File System \\
\hline $\mathrm{Nu}$ & Nusselt Number \\
\hline $\mathrm{OC}$ & Open Compute \\
\hline OR & Open Rack \\
\hline $\mathrm{OU}$ & OpenU, rack unit height, $1 \mathrm{OU}=1.89 "(48 \mathrm{~mm})$ \\
\hline $\mathrm{P}$ & Pressure $\left[\right.$ in. $\left.\mathrm{H}_{2} \mathrm{O}\right]$ \\
\hline $\mathrm{PC}$ & Personal Computer \\
\hline $\mathrm{P}_{\mathrm{C}}$ & Critical Pressure [in. $\mathrm{H}_{2} \mathrm{O}$ ] \\
\hline PCB & Printed Circuit Board \\
\hline PCIe & Peripheral Component Interconnect Express \\
\hline PFC & Passive Flow Curve \\
\hline PI & Partial Integral controller \\
\hline POD & Performance Optimized Datacenter \\
\hline PXE & Preboot Execution Environment \\
\hline Q & Airflow [CFM] \\
\hline $\mathrm{Q}_{0}$ & Operational Airflow [CFM] \\
\hline Q\% & Percentage airflow reduction from FD \\
\hline$\rho$ & Density $\left[\mathrm{kg} / \mathrm{m}^{3}\right]$ \\
\hline $\operatorname{Re}$ & Reynolds Number \\
\hline SEB & SAS Expansion Board \\
\hline$S_{h}$ & Energy source per unit volume \\
\hline SMART & Data from a hard drive or solid state drive's self-monitoring capability \\
\hline SSD & Solid State Drive \\
\hline$T$ & Temperature \\
\hline $\mathrm{T}_{2,1}$ & Absolute temperatures during stressed and normal operations \\
\hline$\tau_{i j}$ & Stress tensor \\
\hline$u_{i}$ & Velocity component in Cartesian direction $i$ \\
\hline $\mathrm{V}$ & Average velocity $[\mathrm{m} / \mathrm{s}]$ \\
\hline VFD & Variable frequency drive \\
\hline
\end{tabular}

\section{Introduction}

Every data hall (data center) is expected to deliver information technology services such as telecom, compute, storage or usually a mix of all three. IT equipment can be divided into three main categories based on their functions: 1- Compute/Web Servers; 2- Network Switches; 3- Storage units (servers). Each category IT equipment has three major requirements to guarantee its functionality: space, power and sufficient cooling. In terms of cooling, IT equipment research has focused on the compute category of IT. This is due to the high frequency central processing unit (CPU), which forms the building block of computing data center infrastructure. This particular focus is justifiable given Moore's law, which relates the increased transistor density with the increase in heat fluxes.

Growth in data storage is being fueled by social media usage and the large volume of images and videos that are being generated from mobile devices, and personal computers (PC). At the same time, machines themselves are generating more data than ever before. An example is the Large Hadron Collider that can generate about $40 \mathrm{~TB}$ of data per second during a simulation of the Big Bang model. Note that even a user's sixty seconds online transaction with a retailer can generate a significant amount of data on the products, prices, payment methods, manufacturer, and credit history involved. Consequently, it is no surprise that worldwide data storage has increased by nearly 8000 Exabytes (EB) from 2005 to 2015 [1].

Data storage can be accomplished with Solid State Drives (SSD), Mechanical Hard Disk Drives (HDD), or Tape. SSDs which do not contain any mechanical parts, store data on interconnected flash memory chips. Mechanical HDDs utilize rotationally spinning magnetic media for data storage. The main difference with SSD is that HDD technology is more mature and of significant lower cost per unit storage which also fits perfectly in applications such as cold 
storage or archive. For HDDs, the power generated in the disk drives arises from mechanical frictional resistance, motor work, and from electronic package heat dissipation. Its primary components are the aluminum die casting (housing), the sheet metal cover, the spindle attached to a motor and several disk shaped platters, and a printed circuit board (PCB) with memory and an application-specific integrated circuit (ASIC) devices to allow for interface and communication between the magnetic media and the external devices and systems. The case and mechanical parts of the HDD are shown Fig. 1. (a), the arm motion is derived through the Lorentz force by passing a current through the coil in the presence of a magnetic field.

Storage servers often place multiple HDDs in close proximity to each other with narrow passages or air gaps between them to facilitate airflow. Historically, these storage server designs place the HDDs at the front of the server so as to allow users to insert and eject the drives with minimal impact to the server's operation during drive replacement. In the pursuit of higher volumetric drive densities in the rack and Open Compute (OC) designs, there are newer server products which incorporate denser configurations in which the hard disk drives fill up most of the volume of the storage server, not just the front access area -as in the legacy storage servers-. In such design, it is important to understand various thermo-fluid effects including the temperature profile of the hard disk drive, interactions between the drive and airflow, and the relationship between the air gap and the system level pressure drop. Note that there is very rare published data on the aerodynamic and thermal interactions of storage unit chassis and the HDDs to different environmental conditions let alone high density (HD) OC storage servers.

The Open Compute Project (OCP) aims to design and share intellectual property on efficient servers and storages (data center hardware in general) for scalable computing. The thermal design of an OC data center is discussed in [2]. The study explained Facebook design of an OC 100\% outside air economization facility that uses evaporative cooling and hot aisle containment (HAC). The storage units are usually accompanied by a web server to manage the storage. Optimizing performance through modeling and measurements for an open compute web server unit is discussed in [3] to reduce effects of thermal shadowing on the downstream processor.

Currently, there is a lack of IT airflow data in the available literature. Such data is crucial to operate the data centers in which there is a perpetual deployment of containment solutions. Note that IT equipment thermal compliance is based on an implicit assumption of a guaranteed free delivery airflow intake. However, the airflow mismatches and imbalances can occur due to one or more of the following reasons when the containment solution is deployed: inherent variable utilization of the IT; practices of increasing set points to save energy; load balancing and virtualization; different IT air systems relative strength stacked in the same containment; redundant or total cooling failure; air filters derating with time; environmental changes during free cooling; maintenance of redundant power lines all; initial DC airflow assumptions at the design stage; presence of physical obstruction at airflow vents; or rack/IT specific reasons (e.g. side intake vents in a narrow rack).

In an effort to produce a library of IT equipment airflow characteristics, Alissa et al. [4] has conducted an experimental analysis on different IT air systems (1U, 2U, 9U compute, blade, and switch). The Active Flow Curve (AFC) method is discussed in detail with its corresponding three regions of airflow. The necessity of IT airflow information such as the free delivery $(\mathrm{FD})$ and critical pressure $\left(\mathrm{P}_{\mathrm{C}}\right)$ is emphasized especially when containment solutions are deployed. The flow regions are also correlated to the processor's temperature.

A comprehensive investigation has been carried out by Sankar et al. [5] to use observations in a production data center to make reliability correlations of failure for HDDs based on IT inlet and facility set point temperatures. This research introduces a reliability model that shows that the failure rate can double at an elevated HDD temperature. Also, the energy efficiency of the facility vs. the availability of the HDDs trade-off is discussed. In the same study it has been shown that HDDs' failure had a 71\% share of the annual failure rate of all the server components.

ASHRAE TC 9.9 has issued a thermal guidelines and best practices whitepaper for storage equipment (Tape, HDD, SSD and PCIe based SSD) in data centers [1]. One of the main findings is that more airflow data is required as a function of utilization, power and temperature. This is even more important when air containment is applied in data centers increasing the possibility of airflow imbalances [4]. A data center, especially with containment, is prone to airflow imbalances due one or more of the following reasons:

1) Inherent Variable Utilization of The IT; plus the likely power on sequencing of CRAHs by the building management system (BMS)

2) Practices of Increasing Set Points to Save Energy;

3) Different IT Air Systems Relative Strength Stacked In The Same Containment;

4) Loss of utility power resulting in all CRAHs being off until generator power provides CRAH power,

5) Loss of chilled water to some or all the CRAHs, such that room temperature increases to the point that IT component air systems go to high speed and all IT components attempt to get maximum design airflow

6) Environmental Changes during Free Cooling 
7) Maintenance of Redundant Power Lines or Derating Air Filters

Consequently, it is important to understand the impact of airflow imbalances on the thermal performance of the storage server as well as the HDD reliability and utilization.

In this article, experimental and numerical analyses are performed to analyze the operation and reliability of open compute (OC) storage in a contained environment during airflow imbalances. First, an OC HD storage unit is analyzed experimentally via the airflowflow chamber. The effects of airflow imbalances on the temperature, reliability, and utilization of the HDDs are discussed and an airflow prediction model is introduced. Second, a computational fluid dynamics model is created for a HD open rack in which the measured airflow responses for the OC IT (storage and compute servers) are used to build compact models and run rack level testing for IT air systems sensitivity. In the third part, cooling failure in a storage POD is modeled using IT fan control system (FCS) with a generic control function and is compared to the simple flow curve model.

\section{OC Open vault storage description}

The Open Vault storage unit [6] is a 2 OpenU (OU) chassis that houses a total of 30 HDD as seen in Figs. 1 $(b, c)$. The dimensions of the chassis are $53.6 \mathrm{~cm}$ (21.1") wide x $8.89 \mathrm{~cm}$ (3.5") high x 87.8 (34.6") deep. It consists of two identical 1OU HDD trays with $15 \times 3.5$ " form factor HDDs and two Serial Attached SCSI (SAS) expander boards on each, one fan control board, and six redundant hot pluggable fan modules (each with two blades to overcome dense HDD array pressure drop) mounted externally at the rear of the chassis. The Open Vault storage server fits into the Open Compute Project's (OCP) Open Rack (OR). Herein, the term open vault, storage unit, and storage server are used interchangeably.

Open vault storage availability is maximized by two main features of the unit:

1-Each SAS expander board and HDD tray can be extracted and serviced independently without impact to the other connected trays. This enables the easy replacement of one SAS expander board or the replacement of a single HDD while keeping the whole system running.

2- Each fan module is hot pluggable and field replaceable from the rear of the chassis.

Herein, (front) refers to the cold aisle side of the chassis, which is where most of the service occurs, and (rear) refers to the hot aisle side of the chassis, which is where which the fan module service occurs. (SEB) refers to the SAS expander board, (DPB) refers to the drive plane board, and (FCB) refers to the fan control board. Note that the storage server unit is always connected to a web server (Windmill) unit as shown in Fig.1 (d).

\section{Experimental setup}

The storage unit is mounted on the flow chamber [4], as shown in Fig. 1. (d) The airflow and external impedances are controlled by the counter blower, a sliding gate valve called a blast gate, and the nozzles array selected. Airflow straighteners are installed upstream and downstream of the nozzles array. The chamber at the intake face of the storage server simulates a cold aisle with variable pressure while the outlet is a pseudo hot aisle at gauge pressure. Through the open vault Intelligent Platform Management Interface (IPMI) the fans' speed is controlled and data is collected for fans' speed, HDD (via SMART) and PCB temperatures, and HDD utilization, in order to detect and locate any throttling activities. The Windmill server which is PXE/NFS, boots from the network into a Debian Jessie environment running Linux 3.16.14. The Windmill server communicates with the storage server; through a LSISAS9207-8e HBA card, HDDs configured in JBOD architecture with no redundancy scheme specified. To equally stress the HDDs, we ran the standard benchmark with the following configuration: random read-writes with a mix of $75 \%$ read operations and $25 \%$ write operations.

In addition to the IPMI/SMART readings, three UAS1000 temperature and velocity sensors (hot wires and thermistors, Fig. 1(c)) with an accuracy of $\pm 0.05 \mathrm{~m} / \mathrm{s}$ and $\pm 1{ }^{\circ} \mathrm{C}$ respectively are allocated at the intake before the HDDs, shown in green circles Fig. 1 (c), and just after the HDDs before the fans, shown in red circles. Readings of these sensors are used to calculate average velocity and temperature readings for Re and $\mathrm{Nu}$ calculations.

\section{Active flow curves (AFC) analysis}

The AFC is a simple representation of the behavior of the chassis' impedance and air system combined. The interaction between fans and the degradation to fan curves are all collapsed in the AFC model. The curves characterize the behavior of IT air systems with external variations of back pressure. The regions of airflow [4] are divided into three, aided, hindered and reversed flows. The free delivery point (FD) is the point at which the external impedance is zero. The critical pressure point $\left(\mathrm{P}_{\mathrm{C}}\right)$ is defined as the point at which the flow through the server intake $\sim 0$ CFM. These two points are unique to the given IT equipment and depend on the internal layout, air system, and fan speed. The formula of the (AFC) [4] is shown in Eq. (1): 
where $\mathrm{K}_{\mathrm{i}}$ and $\mathrm{K}_{\mathrm{v}}$ are modified coefficients that originate from the chassis' internal resistance and are a function of the airflow direction through the chassis [7]. Eq. (1) is analogous to the Darcy-Forchheimer equation for flow pressure drop through porous media with the term $\mathrm{P}_{\mathrm{C}}$ added to represent an additional source within the porous media (i.e. server fans). Fig. 2 and Table 1 show the AFC analysis data for $100 \%, 75 \%$, and $50 \%$ fan speed. The AFC is measured first for $100 \%$ fan speed, for which the FD and $\mathrm{P}_{\mathrm{C}}$ are measured to be $154.55 \mathrm{CFM}$ and $1.01 \mathrm{in}$. $\mathrm{H}_{2} \mathrm{O}$, respectively. Since the affinity laws, Eq. (2), apply to the AFC, the $100 \%$ fan speed curve is used to estimate the airflow and pressure values at the two other speeds as seen in the green and blue dash-dotted curves. To validate the estimated curves, the AFC testing procedure is repeated again for the cases of $75 \%$ and $50 \%$ fan speed as shown in the green and blue circular points. Good agreement is found between the measured points and estimated curves of the AFCs. Note that this method is greatly beneficial to the platform or facility level controls for assessing the cooling needs of each storage unit. The advantage lays in the fact that the airflow can be accurately estimated for each individual storage unit in the aisle. This is of great importance especially in contained solutions where airflow mismatches are plausible. Table 1 compares the measured and estimated FD and $\mathrm{P}_{\mathrm{C}}$ values. Here, it is shown that the error for the airflow is less than $5 \%$ and is less than $10 \%$ for the pressure. (See Inline Supplementary section S-2, Fig. S2, and Fig. S3 for thermal response of the HDD in the regions of the AFC during $100 \%$ fixed fan speed operation).

$\frac{Q_{\text {current }}}{Q_{\text {ref }}}=\frac{N_{\text {current }}}{N_{\text {ref }}}, \quad \frac{P_{\text {current }}}{P_{\text {ref }}}=\left(\frac{N_{\text {current }}}{N_{\text {ref }}}\right)^{2}$

\section{Airflow imbalance test with allowed fans response}

To understand the effect of airflow mismatches that can happen during normal operation or failure modes; a back pressure of 0.12 in. $\mathrm{H}_{2} \mathrm{O}$ is introduced however for a longer duration (transient test) and the response of the storage system is observed under the same read/write job condition. Note that this values was chosen since it corresponds to the critical pressure of the AFC at normal operation at which the fans duty is 33\% of the maximum RPM. In this test, no fan speed constraints are applied. This will allow observing the actual response of the hardware fans' algorithm. The test starts while the chassis is operating at its free delivery airflow with zero external impedance. Then a back pressure perturbation is introduced for $\sim 70$ minutes. During this period the HDDs and PCBs overheat. The FCS (fan control system) responds to that by increasing the fans' speed. Note that in a well-sealed containment this would increases the external impedance further or degrade the surrounding IT with weaker air systems (cascade failure effect). After that, the external impedance is removed, the unit is allowed to recover and the RPM gradually drops to initial value. The PCBs recuperate to their initial steady state temperature, but the HDDs require a much longer steady state time due their higher thermal inertia. The pressure/flow test cycle is shown in Fig. 3 (a). The storage server unit has six fan modules (each module has a front and rear fan rotating in counter directions with seven and five blades per fan for the front and rear fans, respectively). Initially, the fan speeds are at 2500 and 3100 RPM as shown in Fig. 3 (b). When the external impedance is applied the component temperatures increase and fans ramp up based on the FCS until the internal components reach a local steady state. When the system is relived of external impedance the fans gradually drop in RPM. Lower HDDs shelf was observed to have slight higher temperatures than the upper one and so more focus will be made there. By observing the HDDs temperature charts, Fig. 4, the rear HDDs tend to be more susceptible to overheating since the air stream has already passed through the upstream boards and HDDs causing a thermal shadow region at the rear.

\subsection{Impact on utilization}

Bandwidth and IO are correlated to the thermal performance. The total IO is shown in Fig. $5(a)$. for the HDDs. It can be deduced that the rear HDDs, which are deeper, are generally observed to have a lower total IO due to thermal shadowing by the upstream HDDs and boards. The total IO reduction will accumulate to yield bigger differences over longer time intervals. The runtime displays the time interval during which the HDDs are performing a read or write command/request. When the HDDs start to overheat they also start to throttle (processing speed slows down as temperature increases) requests to write or read which explains the reduction in the runtime of the rear thermally shadowed HDDs as shown in Fig. 5 (b). Similarly, for longer durations of airflow mismatches the underutilization effect will accumulate in the OC storage environment. 


\subsection{Impact on reliability}

The failure rate of HDDs in the storage unit due to airflow mismatches can be further investigated by utilizing the Arrhenius acceleration factor (AF) formula [5] as shown in Eq. (3) where $(\mathrm{A}=1.25)$ is a proportional multiplier, $(\mathrm{Ea}=$ $0.464 \mathrm{eV})$ is the activation energy determined empirically, $\left(\mathrm{K}=8.63 \times 10^{-5}\right)$ is the Boltzmann's constant that relates energy at the particle level with temperature observed at macro level and finally $T_{2} \& T_{1}$ which are the absolute temperatures during stressed and normal operations respectively. $\mathrm{T}_{1}$ is used at $36{ }^{\circ} \mathrm{C}$ which was the average temperature of the frontal HDDs. The acceleration factor AF is calculated at the median temperature of each set of HDDs (which includes the rear, middle, and front) as the stress test marches in time.

$A F=A e^{-\frac{E_{a}}{K} \times\left(\frac{1}{T_{2}}-\frac{1}{T_{1}}\right)}$

Table 2 shows the AF values for different locations and time steps. It is observed that at the beginning ( $\mathrm{t}=10$ mins) of the test the rear line of HDDs has the highest initial value of AF at 2.16. The lowest AF value is 1.40 and is reported at the front group of HDDs with only $11.84 \%$ deviation from the normal operation AF value. As the test marches in time the probability of failure is highest for the rear HDDs at an AF of 4.82. Those observations can be utilized when deploying the storage units within a contained solution. The practice of reducing the cooling infrastructure's blower speed to exactly match the IT airflow demand can save a considerable amount of blower power. Yet, at any of the previously mentioned cases the airflow balance can be lost and the AF of the HDDs starts to increase, which endangers the availability of service. The proposed airflow mismatch test produces data and correlations, such as in Eqs. (4-6), that relate facility level events with the life expectancy of storage drives.

$\begin{array}{ll}A F=0.034 t+1.881, & R=0.98 \\ A F=0.026 t+1.586, & R=0.98 \\ A F=0.016 t+1.314, & R=0.96\end{array}$

(4) $($ rear HDDs)

(5) (middle HDDs)

(6) (front $H D D s$ )

\subsection{Impact on server PCB and facility cooling control}

By inspecting the PCBs temperatures as shown in Fig. 6 (a), it can be observed that the Drives plane board average temperature increases by more than $10^{\circ} \mathrm{C}$ during the airflow reduction. For the SAS expander board at the front, the temperature increases from 31 to $45^{\circ} \mathrm{C}$ at a high rate. As the fans modulate to higher speeds, the rate of temperature decreases. However, note that the total steady state may have not been achieved yet. As the fan speed increases the rates of heating will decrease but have not stop indicating that even subtle airflow mismatches have a long term creeping effect in which the components temperatures increases over longer time spans. Ultimately, this will result in inefficient operation of the server (increased power and current leakage) and eventually overheating failures of HDDs or boards. The rates of temperature are also a function of the applications running on the HDDs (pictures, videos, streaming). In this proposed airflow mismatch test, the airflow imbalance effect is accelerated in a thermal stress/cycling tests to show the behavior of the storage unit during airflow mismatches.

When looking at the IPMI ambient sensors data, Fig. $6(b)$., it can be seen that they start at 22 and $27^{\circ} \mathrm{C}$ at the beginning of the test, these values are $\sim 3{ }^{\circ} \mathrm{C}$ higher than the manually installed sensors because of preheat and proximity to hardware. However, the difference between the platform and the external sensors data will increase during the airflow imbalance. A similar observation was reported in [8 \& 9] during facility level air side variations experiment for legacy compute IT equipment in CAC. This is an indication that external discreet sensors were not able to reflect the actual availability of the hardware during the airflow mismatch case. In any kind of servers, the inconsistency between the IPMI inlet temperature sensor and the external inlet sensors is expected to increase in cases of airflow imbalances. This is an indication that using only external sensors for IT intake in data centers can be misguiding especially during cooling failures where it is most critical.

\section{Rack level simulation of the OC storage/Server IT infrastructure}

Thus far, we have discussed the thermal performance of an individual storage unit. However, in a real production data center environment the interactions are much more complex. For an instance, one open compute storage POD will contain OCRs populated with an array of storage servers and Windmill web servers. Prototyping for facility level and sometime rack level can be impossible. The alternative to this is by utilizing computational fluid dynamics simulation (CFD). Simulation has been used in electronics cooling for decades to make critical decisions such as CPU location and selection of blowers or heat sinks. After the design is optimized, the chassis is put to mass production 
maintaining all the specifications that were made by the design team. For the data center rack or facility level the situation is much more intricate. The rack or facility can contain tens or thousands of servers -each server can be considered as a CPU-. The servers are subjected to relocation, replacement/IT refresh and addition. Using this analogy, this is similar to a huge IT chassis with thousands of CPUs that are being relocated, replaced or increased. This dynamic environment requires continuous optimization for the data center or what is termed as operational/continuous modeling. Rack level models are especially important for hardware manufacturers where racks are shipped to cloud service providers already equipped with IT. Herein, the 3D Reynolds-averaged Navier-Stokes (RANS) and energy equations are discretized and solved using the finite volume scheme [12, 18, and 19];

$$
\begin{aligned}
& \frac{\partial \rho}{\partial t}+\frac{\partial}{\partial x_{i}}\left(\rho u_{i}\right)=0 \\
& \frac{\partial}{\partial t}\left(\rho u_{i}\right)+\frac{\partial}{\partial x_{i}}\left(\rho u_{i} u_{j}\right)=-\frac{\partial P}{\partial x_{i}}+\frac{\partial \tau_{i j}}{\partial x_{j}}+F_{i} \\
& \frac{\partial}{\partial t}\left(\rho C_{p} T\right)+\frac{\partial}{\partial x_{i}}\left(\rho u_{i} C_{p} T\right)=-\frac{\partial}{\partial x_{i}}\left(K \frac{\partial T}{\partial x_{j}}\right)+S_{h}
\end{aligned}
$$

the $k-\varepsilon$ model is used to simulate the turbulent flow. Rack level analysis of the response of the IT (storage and compute) during airflow imbalance will be addressed.

The open compute rack (OCR) consists of three power zones [10]. Each power zone has $10 \mathrm{OU}$ for IT (storage or compute) and $3 \mathrm{OU}$ for the power shelf. Each power shelf provides $4.2 \mathrm{~kW}$ of continuous maximum load via three bus-bar pairs (+/-) with a $12 \mathrm{~V}$ distribution per power zone. The IT is equipped with a clip assembly so they are directly plugged to the open rack bus-bars. Shelfs can be used instead of the conventional mounting rails to support the IT. The rack is modeled in details as shown in Fig. 7 (b) -shelfs are removed when IT are deployed-. A typical storage OCR will house a combination of storage units and web servers. The arrangement can be a function of many parameters such as the redundancy and performance. For the 40 OU open rack; 4 storage servers and 3 Windmill are deployed in each power zone as shown in Fig. 7 (d). In total the open rack contains 12 storage units, 9 web servers and 3 power shelfs. The rack can support $1080 \mathrm{~TB}$ of storage capacity distributed over 360 3TB HDDs in the storage units. Compact IT models also called black box or simplified server models (SSM) are required for larger scale simulation [11\&12]. The airflow characteristics of the IT can be extracted from detailed models, Fig. 7 (c) or through direct measurements -which is done here-. A compact model is created for the open vault and Windmill in which the measured passive and active airflow characteristics are applied as boundary conditions, the vent sizes were matched to actual IT to account for the jet throw distance. Since the fans were located in series, each vent locations will be assigned with a portion of the total AFC curve that is one sixth of the AFC airflow for the open vault and half for the Windmill per vent. The open vault and the Windmill dissipate $600 \mathrm{~W}$ and $237 \mathrm{~W}$ of heat respectively and are cooled via forced air ventilation. Based on fan speed measurements during the experimental part, the FCS dictates a fan speed of $\sim 33 \%(\mathrm{FD}=51 \mathrm{CFM})$ and $18 \%$ ( $\mathrm{FD}=$ of maximum speed for the open vault and Windmill respectively during normal operation, so the AFC is adjusted using affinity laws and applied as a boundary condition(see Inline Supplementary Fig. S5 for Windmill AFC test). The power shelf hardware is not available for experimental characterization and spec data are assigned with a power of $200 \mathrm{~W}$ at the assumption of $120 \mathrm{CFM} / \mathrm{kW}$ airflow to power ratio. In summary, at the current fan speed, the rack level free delivery is $\sim 920$ CFM and the heat dissipated is $9.93 \mathrm{~kW}$. As shown in Fig. 7 (d), K1-K12 designate the open vault storage units starting from bottom to top, similarly, W1-W9 refer to the Windmill server units.

The rack test chamber is a prototype data center environment where the airflow is supplied from the raised floor and exhausted through the upper right vent, Fig. 7 (a). The test chamber is $(2.1 \mathrm{~m} \times 0.6 \mathrm{~m} \mathrm{x} 2.4 \mathrm{~m})$ and the model is resolved with a $91 \mathrm{~K}$ structured grid cells. This scheme can be used as a generic containment configuration. The rack level IT air systems interaction to airflow imbalances will be analyzed between the compute and the storage servers. Three pressure sensors are located at the front and rear of the rack, the average reading is taken at each side and pressure differentials are obtained $\left(\mathrm{P}_{\text {rear }}-\mathrm{P}_{\text {front }}\right)$. Different levels of airflow imbalances are simulated and plotted against the percentage airflow reduction from free delivery $\mathrm{Q}_{\%}$. Fig. S4 (a) (see Inline Supplementary Fig. S4). Presents the airflow reductions for the open vault (storage) units namely $\mathrm{K} 4, \mathrm{~K} 8$ and $\mathrm{K} 12$ bottom to top. Between pressure values -0.03 and 0 in. $\mathrm{H}_{2} \mathrm{O}$ the storage servers are overprovisioned with cold air supply and experience an increase in the operating airflow up to $40 \%$ higher than FD (note that the negative signs for the pressure differentials and Q\% depend on the definition). It is noted as well that $\mathrm{K} 12$ experiences the highest increase of airflow during overprovisioning since the pressure build up is relatively higher at the top because of airstream stagnation. When the system is underprovisioned, the pressure values are between 0 and 0.045 in. $\mathrm{H}_{2} \mathrm{O}$. The storage units experience a reduction from the 
FD up to $\sim 39 \%$ of which the reliability impact was addressed in the experimental testing sections. Note that the inlet conditions of the rack or facility level models can used as inputs to the experimentally obtained reliability and internal temperature correlations of HDDs or boards. This is one of the main advantages of using IT level characterization into compact models for rack or data center simulations as it creates a link between the facility and vital IT internal components. Fig. S4 (b) (see Inline Supplementary Fig. S4) displays the Windmills' airflow variations under the same external impedance conditions. The main observation is that they show higher sensitivity to airflow imbalances. For an instance, at 0.042 in $\mathrm{H}_{2} \mathrm{O}$ the airflow reduction is $\sim 80 \%$ but less than $40 \%$ for the storage units. Bearing in mind that the main active components in the Windmills are CPUs and DRAMs with much higher frequency than HDDs and hence more prone to throttle or overheat. The relationship between CPU temperature and airflow mismatches was previously shown in [4]. Advanced IT and data center simulation tools can model the response of the FCS to the IT inlet temperature using temperature dependent AFCs where each inlet temperature is assigned with a certain fan speed value. However, there is still a need for identifying the response of the FCS upon airflow reduction. This gets more complicated since overheating would be more location specific inside the server, and hence, the FCS response would be different from one system to another since IT products might defer in defining the critical temperatures inside.

The application of the AFC for airflow prediction per IT using fan speed and pressure input was discussed in previous sections. The accurate knowledge of the airflow also aids in avoiding overcooled facilities that were designed on maximum values or conservative assumptions of airflow to power ratios. However, at certain conditions there exist internal impedances within the rack itself such as in the RDHX case. Pressure measurements can be more challenging there between the IT inlet and outlet vents. We can utilize the rack level model however to obtain a total rack level AFC as shown in Fig. S4 (c) (see Inline Supplementary Fig. S4). Eqs. (10-12) represent the linear regression of the data at an average rack fan speed (ARFS) of $25 \%$. This formula can be used for rack level airflow prediction where $\alpha$ is a correction factor for higher IT fan speed and is calculated by averaging the IT numbers and type.

$Q=\alpha \times(-12843 \times P+996.61)$

$\alpha=\frac{A R F S_{\text {current }}}{A R F S_{\text {ref }}}, \quad A R F S_{\text {current }} \geq A R F S_{\text {ref }}$

ARFS $=\frac{\sum_{i=\text { type } 1,2, \ldots . .}^{n}(\text { IT Count } \times N)_{i}}{\sum_{i=\text { type } 1,2, \ldots . .(\text { IT Count })_{i}}^{n}}$

\section{POD level simulation with IT fan control system}

Modeling of IT equipment airflow has evolved overtime in pursuit of more realistic representation of servers. Initially IT models were based on a fixed airflow quantity dictated by the power generation and temperature difference.

As cooling solutions started to create pressure drop sources around the IT such containment or in cabinet heat exchangers, the fixed airflow model failed to yield the accurate representation of the pressure drop effects. Consequently, another model arose that utilizes the server's total fan curve intersected with its impedance. This is usually done by combining both curves inside a cuboid that represents the server chassis, and the point of intersection is called the operating point. However, this model has two deficiencies. First the intersection of the total fan curve and the impedance curve can result in significant over estimation of the IT airflow operating point (FD) [20]. This happens because fan curves are obtained using airflow chamber testing while being free of upstream and downstream obstructions which yield in an optimum fan curve. The general rule of thumb is to have one-half diameter of the fan clear upstream and downstream [21] which is not always the case with many servers. The second point is related to high computational expense caused by flow fields resolved inside the cuboid especially when thousands of servers are to be modeled in the facility.

The simplified server model (SSM) mentioned earlier represents the server air system equation/curve as a boundary condition on the vent. The black box representation enables running simulations with thousands of servers in much shorter simulation time. The AFC is applied at the IT vents after being extracted through testing [4].

In reality, pressure/flow correlations are not sufficient to yield the exact behavior of IT. The reason behind that is the absence of integrated fan control systems (FCS). The server's fan duty cycle vary based on the inlet temperature, component utilization/temperature, and airflow allowed. In this section we will discuss Temperature-Pressure-Airflow curves and how does that affect simulation results in a case of increased inlet temperature.

Fig. 8 shows a numerical test bed of a storage POD that consists of 20 racks having the same IT deployment described previously. Two CRAHs provide cooling to the POD which sits on a $0.91 \mathrm{~m}(3 \mathrm{ft})$ raised floor. Cold aisle 
containment (CAC) is applied and sized to the racks with ideal sealing except for allowed leakage through a $5 \mathrm{~cm}(2$ in) under rack gap which usually induced by rack legs. Details on leakage locations, modeling, meshing, and ranking were addressed in [23]. The POD architecture (without the IT) is based on Binghamton data center laboratory model which analyzed in [20, 23, and 24]. The POD consists of $300 \mathrm{~K}$ cells with increased mesh density near the leakage location [23].

Cross section line A-A slices the POD showing the inlets of the left row of rack as shown in Fig. $8(a, b)$. The racks are supplied with chilled cold air via 18 perforated tiles with $65 \%$ openness as shown in Fig. 8 (c). The raised floor grid (solid or opened tiles) is $0.61 \times 0.61 \mathrm{~m}$ per tile. The POD has a power density of $\sim 2.8 \mathrm{~kW} / \mathrm{m}^{2}$ resulting of heat dissipated by 500 different pieces of equipment.

The POD is cooled by two CW114 CRAH units supplying chilled air at $20^{\circ} \mathrm{C}$. Both cooling units have a Variable frequency drive (VFD) mechanism by which the airflow rate is adjusted. The CRAHs are divided into a master and slave control strategy where the controller is attached to the master unit and the slave unit follows and both have similar airflow responses. The VFD controller algorithm is based on the pressure differential between the CAC and the room and the signal is processed through a simple Partial Integral (PI) controller that adjusts the CRAH airflow based on the pressure input [12]. A differential pressure set point of $2 \mathrm{~Pa}$ is selected to ensure efficient operation of the containment and a dead band of $1 \mathrm{~Pa}$ is given to the controller. The locations of the pressure differential sensors are shown in Fig. $8(d)$. Both cooling units have a maximum airflow capacity of 13800 CFM $\left(6.51 \mathrm{~m}^{3} / \mathrm{s}\right)$. This means that the redundancy scheme based on IT normal operation (storage at $30 \%$ and compute at $18 \%$ of fan duty) is N+50\% which is also equivalent to $\mathrm{N}+5$ in a data center with 15 cooling units.

In this section we will exercise a case of chiller failure were the CRAHs supply air temperature will increases based on the airflow rates and the system thermal inertias which are the cooling coils and IT mass [7, 24, 25]. However, our focus here is to run the failure scenario using two different IT airflow models. The first model will utilize the AFC data collected for the IT at normal operation regardless of the increase in the inlet temperature. While the second model will utilize a generic fan control function as shown in Fig. 9 (a). As the figure illustrates, the fans duty will change with increased inlet temperature shifting the IT airflow operation to new AFCs. That results in a significant increase in the IT airflow to power ratio as shown in the figure.

The transient simulation is 80 seconds long with a uniform time step of 5 seconds. As shown in Fig. 9 (c), chiller failure starts at $\mathrm{t}=0 \mathrm{~s}$ causing an increase in the supply air temperature from the CRAH. Fig. 9 (b) shows the master CRAH airflow (VFD) response as well as the pressure differential sensors. Obviously with the lack of the FCS model for IT, their airflow demand will not change as the supply temperature increases from the CRAH unit -Fig. 9 (c). Consequently, the pressure differential will maintain the value of the VFD set point and CRAH airflow will not change.

On the other hand, when the IT FCS is incorporated into the failure simulation the airflow demand increases with the inlet temperature causing pressure imbalances in the CAC. This is reported by the differential pressure sensors that urge the CRAH's VFD to operate at higher speed to supply more airflow volume until the CRAH reaches its maximum airflow capacity.

Fig. 10 shows the IT inlet temperature at cross section A-A where $18-27^{\circ} \mathrm{C}$ is the recommended range and $27-32$ ${ }^{\circ} \mathrm{C}$ is the allowable range for shorter durations and at $\mathrm{T}>32{ }^{\circ} \mathrm{C}$ equipment are prone to overheat and shutdown. It can be seen that modeling the IT FCS with the CRAH VFD enables real life simulation of the dynamic between them. Fig. 10 (a) shows that until $\mathrm{t}=40 \mathrm{~s}$ after chiller failure, all IT are still with the recommended range. However when the IT FCS is modeled, Fig. 10 (b) shows that almost all the IT are already in the allowable range. Similarly, at $\mathrm{t}=75$ s Fig. $10(a)$ shows that IT are still in the allowable range while Fig. $10(b)$ shows that about $70 \%$ of IT have already overheated. The airflow imbalance that was captured by the IT FCS model is the main reason for this contrast between the two figures since it induced hot air recirculation through the under rack leakage. This behavior will vary from one POD to another based on the type of containment, leakage location, and IT heat dissipation [14].

\section{$8 \quad$ Concluding remarks}

This study discusses experimental and numerical test methods to characterize the operation and reliability of an open compute storage system in containment where airflow imbalances are plausible. First, an experimental analysis for HD 3D array storage unit is carried out and thermal/utilization responses during airflow imbalances are reported during proposed stressing tests. An IT level airflow prediction model is discussed for the storage unit and validated for different fan speeds. Second, a computational fluid dynamics model is created for a high density open rack in which the measured airflow responses for the open compute IT (storage and compute servers) are used to build compact models, run rack level testing for IT air systems sensitivity, and create a rack level AFC airflow demand prediction model. The experimental characterization data and the rack level model are used to build CAC POD level model that incorporates IT fan control systems (FCS). This modeling approach yields shorter uptime during chiller 
failure due to increased recirculation induced by increased IT airflow demand during cases such as chiller failure or high economizer temperatures.

This study provides some important information for storage IT airflow characteristics, modeling, and reliability in containment; specifically:

1) Comparison of the system impedance between legacy frontal storage and open compute volumetric storage. The latter produced lower system impedance mainly due to the lack of power supply blocks (see Inline Supplementary section S-1 and Fig. S1).

2) A validated dynamic airflow prediction model and testing methodology for the storage server based on the fans RPM and facility level simple pressure measurements. This method is beneficial to the platform or facility level controls for assessing the cooling needs of each storage unit.

3) Summarizes reasons that induce airflow mismatches for data center.

4) Discusses experimental reliability tests for storage server simulating cases of airflow imbalances. The tests show the impact on HDD failure rate as well as reduction in read/write utilization (i.e. IO and runtime).

5) The increased discrepancy of the IT inlet temperature reading between best practices based (external) and IPMI based (internal) measurements during cases of airflow imbalances.

6) Utilizing experimentally obtained characterization data to build a simplified rack level airflow prediction model that is IT arrangement specific.

7) Utilizing inlet temperature dependent AFC to model the variation of the IT fan control system under different inlet temperatures and varying pressure controlled cooling unit airflow supply.

Generally speaking, IT inlet temperature is only one of the factors that determines the behavior of IT airflow system. The airflow imbalance test has shown that when the inlet airflow is restricted the fan controller quickly ramps up the fan speed to compensate. The inlet air temperatures however remain constant because they are not flow rate related. This is important because predictive modelling cannot reflect this behavior since currently data center CFD IT models have nothing to reflect the impact on component temperature. Furthermore, aisle containment creates an environment where substantial pressure differences can be created by flow imbalances such as might result from a redundant cooling unit failure. This intern could result in an unexpected cascade failure if designers/planners cannot predict the behavior in design/planning and modify the design to compensate. Future research will focus on creating a simplified compact model that can predict an indicative internal temperature that will drive the control behavior and predict the appropriate flow rate for any given steady or transient flow condition.

\section{Acknowledgment}

We would like to acknowledge Prof. Kanad Ghose, Kevin D Hall from Binghamton University-ES2 Center Computer Science and Data Center group. We also like to acknowledge Dr. Veerendra Mulay, Dr. Mike Yan, Peter Ha, and Song Liu from Facebook, Inc for their support. We also like to thank Dr. Madhusudan Iyengar from Google Inc. for his insightful suggestions during the inception of this project. This work is supported by NSF I/UCRC Award No. IIP-1134867 and MRI Award No. CNS1040666.

\section{References}

[1] ASHRAE Technical Committee 9.9, Data Center Storage Equipment-Issues and Best Practices. Atlanta, GA, USA: ASHRAE Publications, 2015.

[2] Frachtenberg, E., Lee, D., Magarelli, M., Mulay, V., \& Park, J. (2012, May). Thermal design in the open compute datacenter. In Thermal and Thermomechanical Phenomena in Electronic Systems (ITherm), 2012 13th IEEE Intersociety Conference on (pp. 530-538). IEEE.

[3] Mani, D., Fernandes, J., Eiland, R., Agonafer, D., \& Mulay, V. (2015, March). Improving ducting to increase cooling performance of high-end web servers subjected to significant thermal shadowing-an experimental and computational study. In Thermal Measurement, Modeling \& Management Symposium (SEMI-THERM), 2015 31st (pp. 319-323). IEEE.

[4] Alissa, H.A.; Nemati, K.; Sammakia, B.G.; Schneebeli, K.; Schmidt, R.R.; Seymour, M.J., "Chip to Facility Ramifications of Containment Solution on IT Airflow and Uptime," in Components, Packaging and Manufacturing Technology, IEEE Transactions on , vol.PP, no.99, pp.1-12, 2016.

[5] Sankar, S., Shaw, M., \& Vaid, K. (2011, June). Impact of temperature on hard disk drive reliability in large datacenters. In Dependable Systems \& Networks (DSN), 2011 IEEE/IFIP 41st International Conference on (pp. 530537). IEEE.

[6] Yan, M. and Ehlen, J., Open Vault Storage Hardware V0.5, Facebook, 2012. 
[7] Alissa, H., A., Nemati, K., Sammakia, B. G., Seymour, M. J., Tipton, R., Wu, T., Schneebeli, K., (2016, May). On Reliability and Uptime of IT in Contained Solution. In Thermal and Thermomechanical Phenomena in Electronic Systems (ITherm), 2016 IEEE Intersociety Conference IEEE.

[8] Alissa, H. A.; Nemati, K.; Sammakia, B. G.; Seymour, M. J.; Tipton, R.; Mendo, D.; Demetriou, D. W.; Schneebeli, K., " Chip to Chiller Experimental Cooling Failure Analysis of Data Centers Part I: Effect of Energy Efficiency Practices," in Components, Packaging and Manufacturing Technology, IEEE Transactions, 2016.

[9] Alissa, H., A., Nemati, K., Sammakia, B. G., Seymour, M. J., Tipton, R., Wu, T., Schneebeli, K., (2016, May). On Reliability and Uptime of IT in Contained Solution. In Thermal and Thermomechanical Phenomena in Electronic Systems (ITherm), 2016 IEEE Intersociety Conference IEEE.

[10] Ning, J., Intel Server in Open Rack Hardware v0.3, OCP, 2013.

[11]King, D., Ross, M., Seymour, M., \& Gregory, T. (2014, March). Comparative analysis of data center design showing the benefits of server level simulation models. In Semiconductor Thermal Measurement and Management Symposium (SEMI-THERM), 2014 30th Annual (pp. 193-196). IEEE.

[12] 6Sigma 9.8 User Manual, Future Facilities, San Jose, CA, USA, 2015.

[13] Cengel, Y. A., Klein, S., \& Beckman, W. (1998). Heat transfer: a practical approach (Vol. 141). New York: McGraw-Hill.

[14] Nemati, K., Alissa, H. A., Murray, B., Sammakia, B. (2016, May). Experimental Characterization of a Rear Door Heat Exchanger with Localized Containment. In Thermal and Thermomechanical Phenomena in Electronic Systems (ITherm), 2016 IEEE Intersociety Conference IEEE.

[15] Nemati, K., Alissa, H. A., Murray, B., Sammakia, B. (2016, May). Steady-state and Transient Comparison of Cold and Hot Aisle Containment and Chimney. In Thermal and Thermomechanical Phenomena in Electronic Systems (ITherm), 2016 IEEE Intersociety Conference IEEE.

[16] Fakhim, B., Behnia, M., Armfield, S. W., \& Srinarayana, N. (2011). Cooling solutions in an operational data centre: A case study. Applied thermal engineering, 31(14), 2279-2291.

[17] Ham, S. W., \& Jeong, J. W. (2015). Impact of aisle containment on energy performance of a data center when using an integrated water-side economizer. Applied Thermal Engineering.

[18] Semin, V., Bardsley, M., Rosten, O., \& Aldham, C. (2015, March). Application of a multilevel unstructured staggered solver to thermal electronic simulations. In Thermal Measurement, Modeling \& Management Symposium (SEMI-THERM), 2015 31st (pp. 287-292). IEEE.

[19] Launder, B. E., \& Spalding, D. B. (1974). The numerical computation of turbulent flows. Computer methods in applied mechanics and engineering,3(2), 269-289.

[20] Alissa, H. A., Nemati, K., Sammakia, B., Seymour, M., Schneebeli, K., \& Schmidt, R. (2015, July). Experimental and Numerical Characterization of a Raised Floor Data Center Using Rapid Operational Flow Curves Model. InASME 2015 International Technical Conference and Exhibition on Packaging and Integration of Electronic and Photonic Microsystems collocated with the ASME 2015 13th International Conference on Nanochannels, Microchannels, and Minichannels (pp. V001T09A016-V001T09A016). American Society of Mechanical Engineers.

[21] Fan Tester, Instruction Manual for AMCA 210-99 Airflow Test Chamber.

[22] Iyengar, M., Schmidt, R. R., Hamann, H., \& VanGilder, J. (2007, January). Comparison between numerical and experimental temperature distributions in a small data center test cell. In ASME 2007 InterPACK Conference collocated with the ASME/JSME 2007 Thermal Engineering Heat Transfer Summer Conference (pp. 819-826). American Society of Mechanical Engineers.

[23] Alissa, H., Nemati, K., Sammakia, B., Ghose, K., Seymour, M., King, D., Tipton, R., (2015, November). Ranking and Optimization Of CAC And HAC Leakage Using Pressure Controlled Models. In ASME 2015 International Mechanical Engineering Congress and Exposition. American Society of Mechanical Engineers, Houston, TX.

[24] Alissa, H. A., Nemati, K., Sammakia, B., Ortega, A., King, D., Seymour, M., \& Tipton, R. (2015, July). Steady state and transient comparison of perimeter and row-based cooling employing controlled cooling curves. InASME 2015 International Technical Conference and Exhibition on Packaging and Integration of Electronic and Photonic Microsystems collocated with the ASME 2015 13th International Conference on Nanochannels, Microchannels, and Minichannels (pp. V001T09A017-V001T09A017). American Society of Mechanical Engineers.

[25] Pardey, Z. M., Demetriou, D. W., Erden, H. S., VanGilder, J. W., Khalifa, H. E., Schmidt, R. R., "Proposal for Standard Compact Server Model for Transient Data Center Simulation," ASHRAE Trans., 2015. 

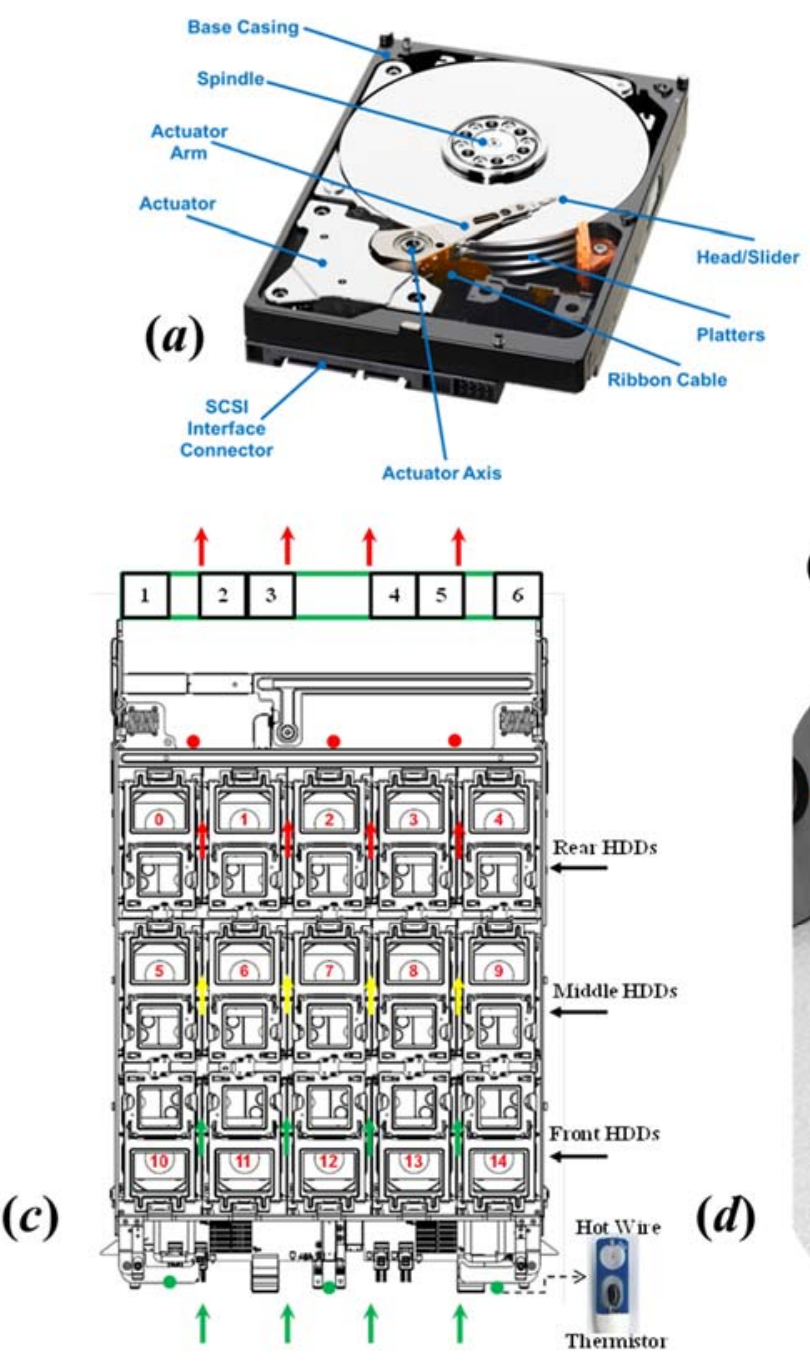

(b)
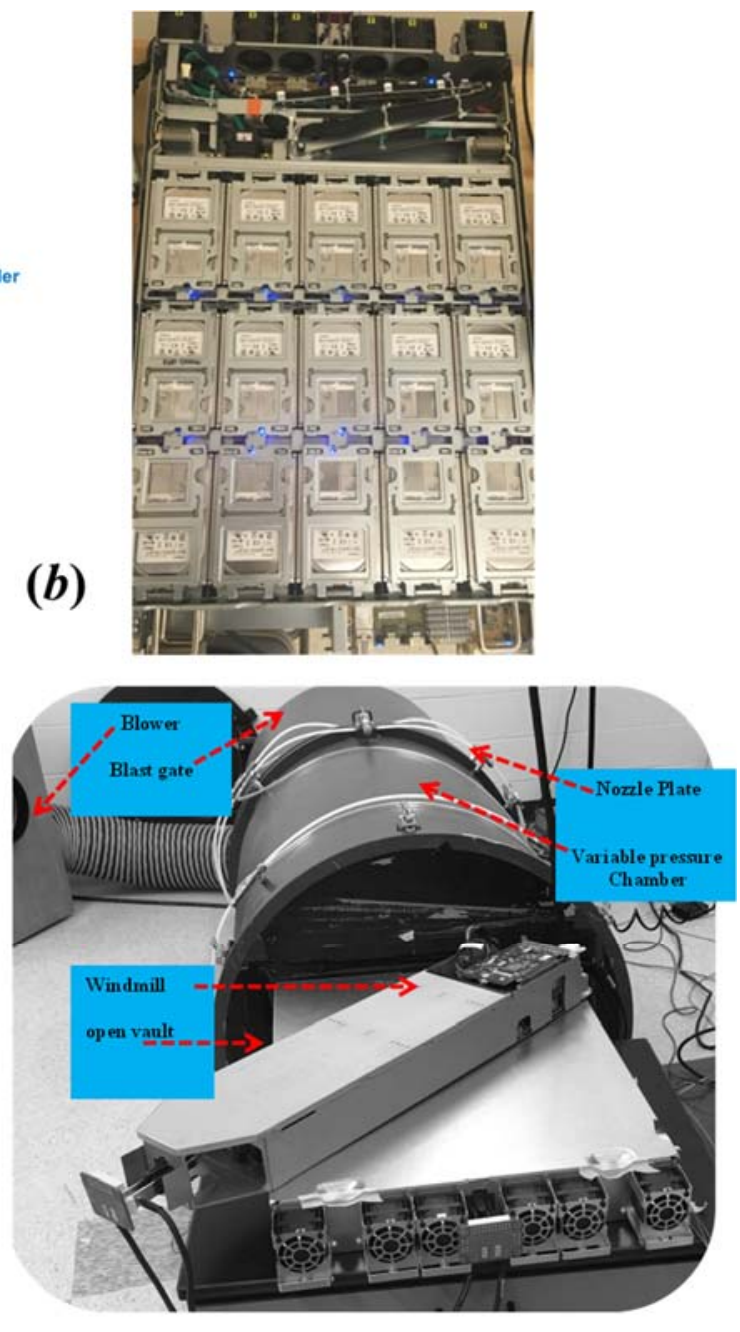

Fig. 1 HDD internal components (b) open vault high density HDD array (c) top wireframe view of the open vault showing HDDs, sensors and fan modules layout (d) flow chamber test setup. 


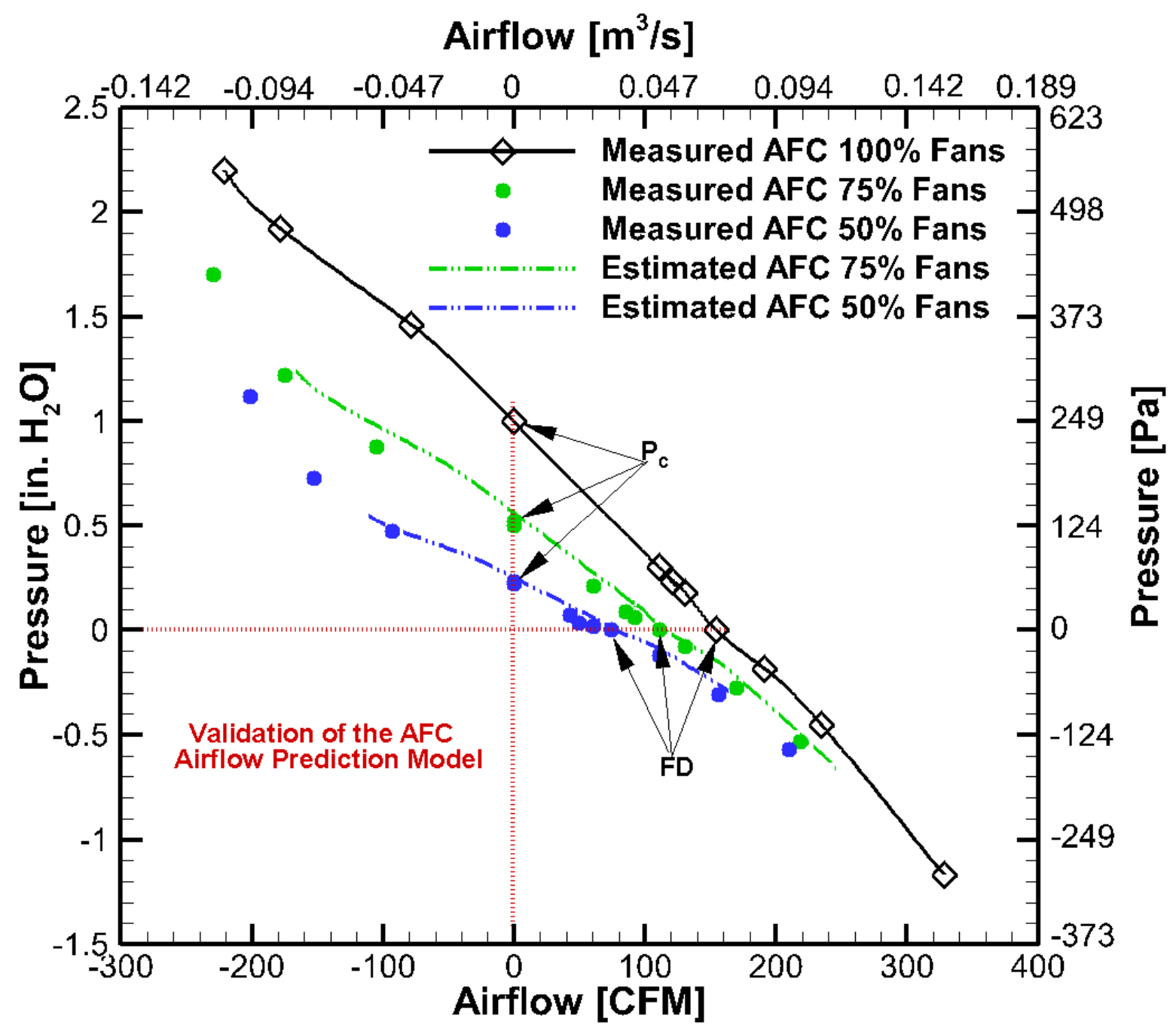

Fig. 2 open vault measured and estimated AFC response under different operating fan speeds (duty cycles).

Table 1

\begin{tabular}{lccc}
\hline \multicolumn{1}{c}{ Air system Property } & $100 \%$ Fans & $75 \%$ Fans & $50 \%$ Fans \\
\hline FD Measured [CFM] & 154.55 & 110.67 & 74.24 \\
$\mathrm{P}_{\mathrm{C}}$ Measured [in. $\left.\mathrm{H}_{2} \mathrm{O}\right]$ & 1.01 & 0.50 & 0.22 \\
FD Estimated [CFM] & -- & 115.91 & 77.27 \\
$\mathrm{P}_{\mathrm{C}}$ Estimated [in. $\left.\mathrm{H}_{2} \mathrm{O}\right]$ & -- & 0.55 & 0.24 \\
$\mid$ FD Estimation Error] & -- & $4.73 \%$ & $4.08 \%$ \\
$\mid \mathrm{P}_{\mathrm{C}}$ Estimation Error] & -- & $10.00 \%$ & $9.09 \%$ \\
\hline
\end{tabular}



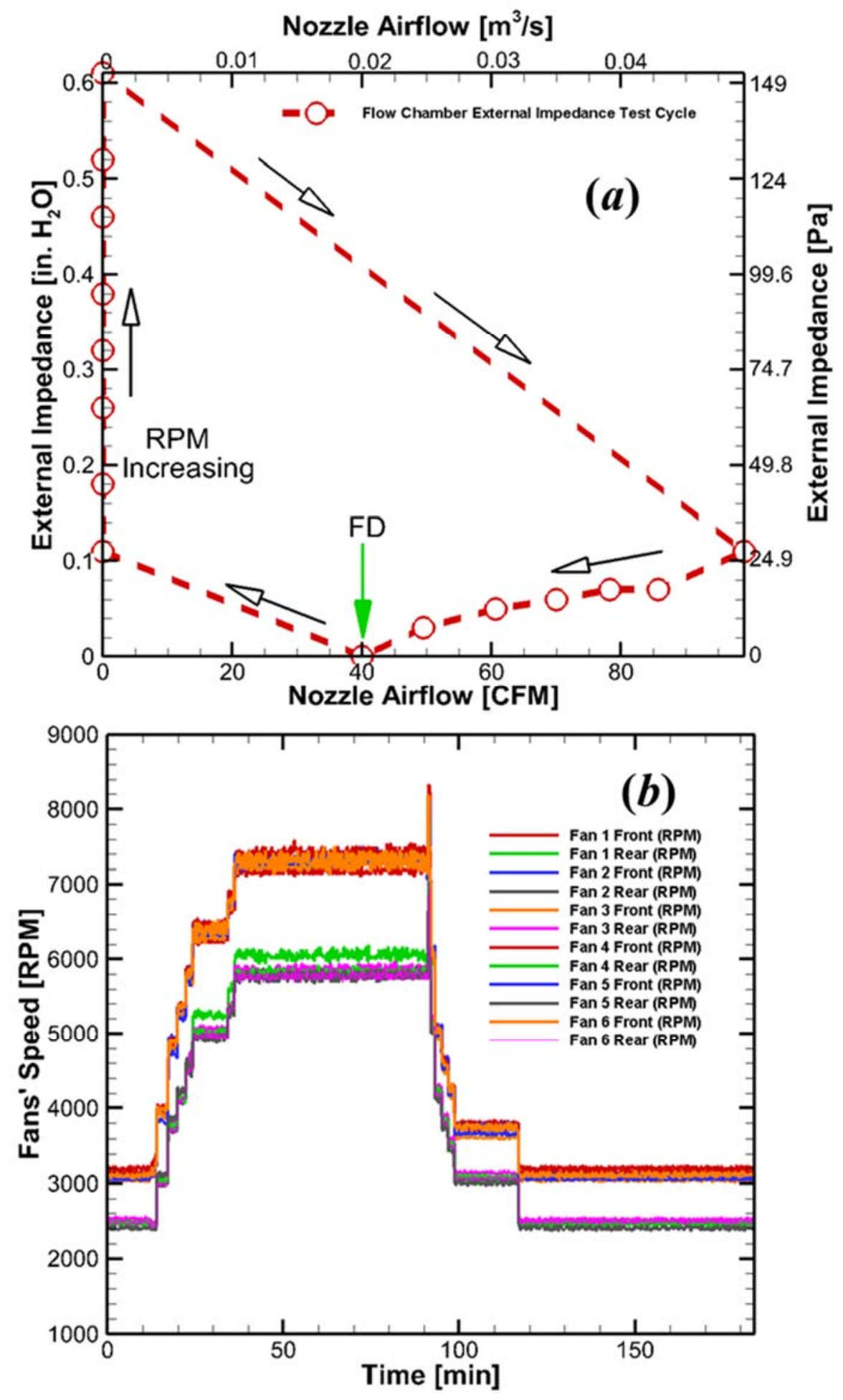

Fig. 3 (a) Pressure/flow cycle of test 2 (b) RPM response during test 2. 

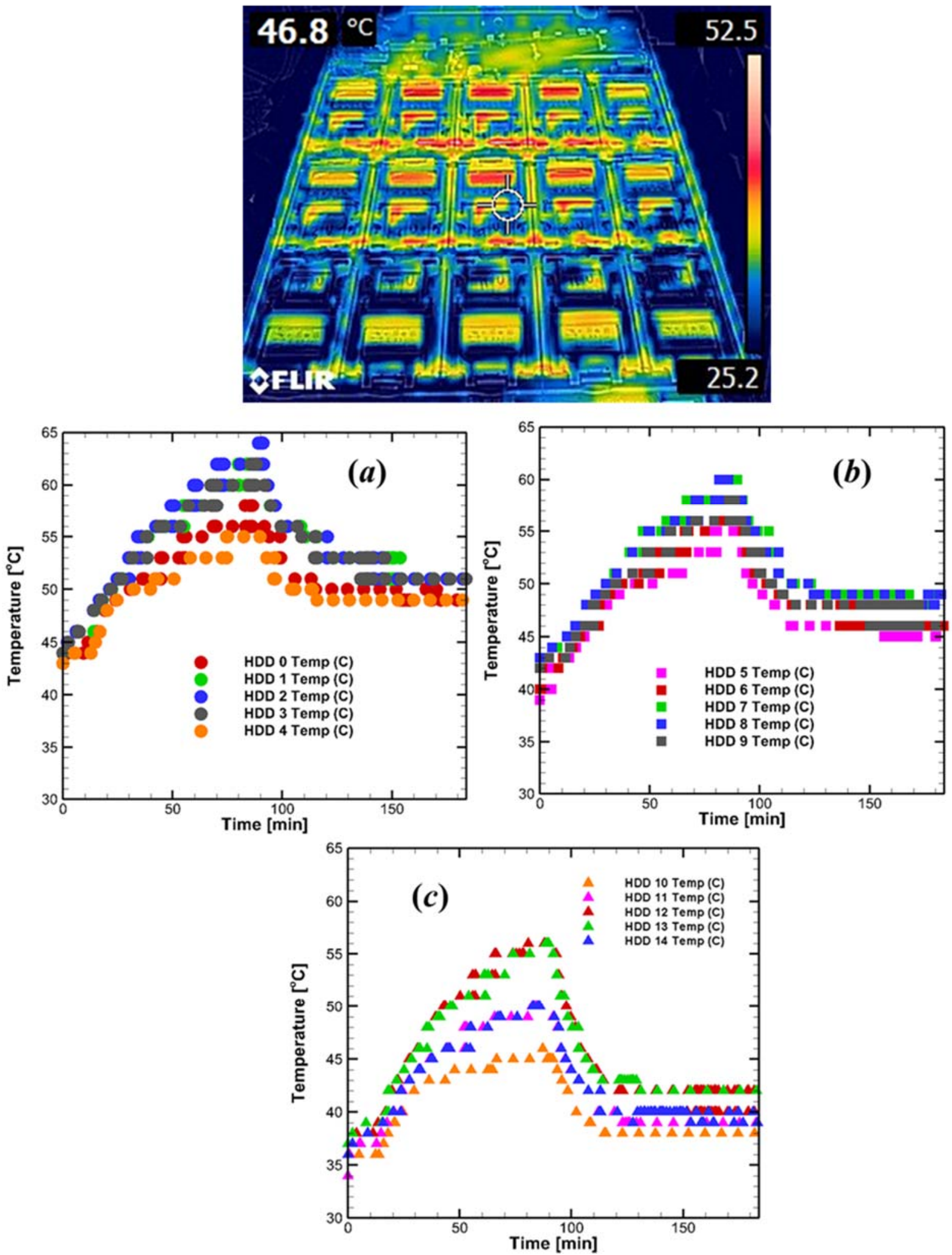

Fig. 4 Rear, middle and front HDD rows SMART temperature. 


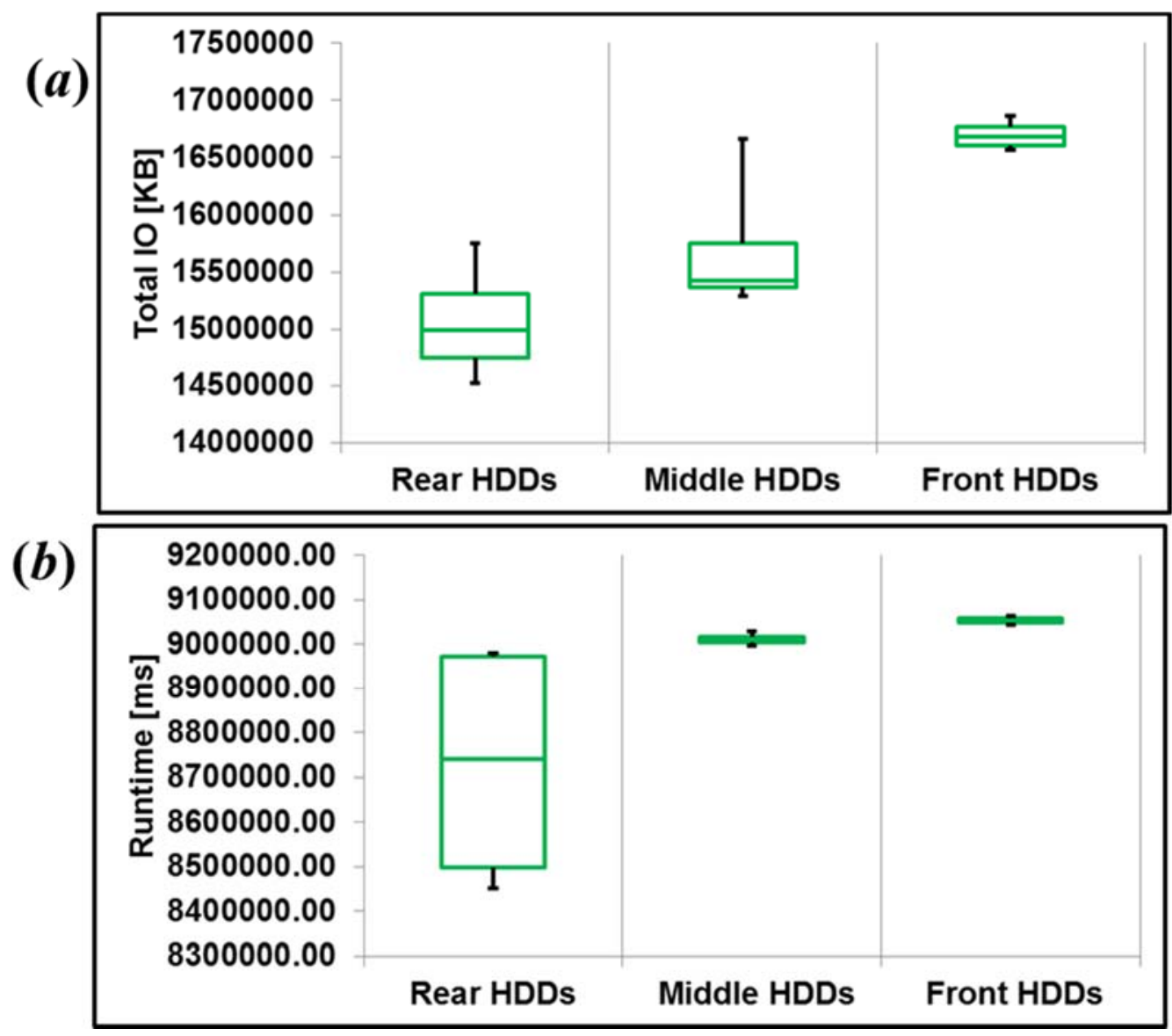

Fig. 5 Distribution of (a) total IO (b) runtime for the rear, middle and front HDDs 
Table 2. Arrhenius model for estimating Acceleration Factor during transient airflow mismatch test [Test 2].

\begin{tabular}{|c|c|c|c|c|c|c|}
\hline $\begin{array}{l}\text { Time } \\
{[\mathrm{mins}]}\end{array}$ & $\begin{array}{c}\text { Rear } \\
\text { HDDs AF }\end{array}$ & $\begin{array}{c}\text { Relative to AC } \\
\text { (a) } 36{ }^{\circ} \mathrm{C}\end{array}$ & $\begin{array}{c}\text { Middle } \\
\text { HDDs AF }\end{array}$ & $\begin{array}{c}\text { Relative to } \mathrm{AC} \\
\text { (a) } 36{ }^{\circ} \mathrm{C}\end{array}$ & $\begin{array}{c}\text { Front } \\
\text { HDDs AF }\end{array}$ & $\begin{array}{c}\text { Relative to AC } \\
\text { (a) } 36{ }^{\circ} \mathrm{C}\end{array}$ \\
\hline 10.00 & 2.16 & $72.54 \%$ & 1.84 & $47.03 \%$ & 1.40 & $11.84 \%$ \\
\hline 20.00 & 2.52 & $101.88 \%$ & 2.05 & $63.63 \%$ & 1.56 & $24.90 \%$ \\
\hline 30.00 & 2.80 & $123.79 \%$ & 2.52 & $101.88 \%$ & 1.84 & $47.03 \%$ \\
\hline 40.00 & 3.42 & $173.99 \%$ & 2.66 & $112.59 \%$ & 2.05 & $63.63 \%$ \\
\hline 50.00 & 3.60 & $187.99 \%$ & 2.80 & $123.79 \%$ & 2.16 & $72.54 \%$ \\
\hline . & • & • & - & - & - & - \\
\hline - & - & - & - & - & - & - \\
\hline . & • & 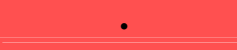 & $\cdot$ & • & • & • \\
\hline 90.00 & 4.82 & $285.91 \%$ & 3.97 & $217.88 \%$ & 2.66 & $112.59 \%$ \\
\hline
\end{tabular}



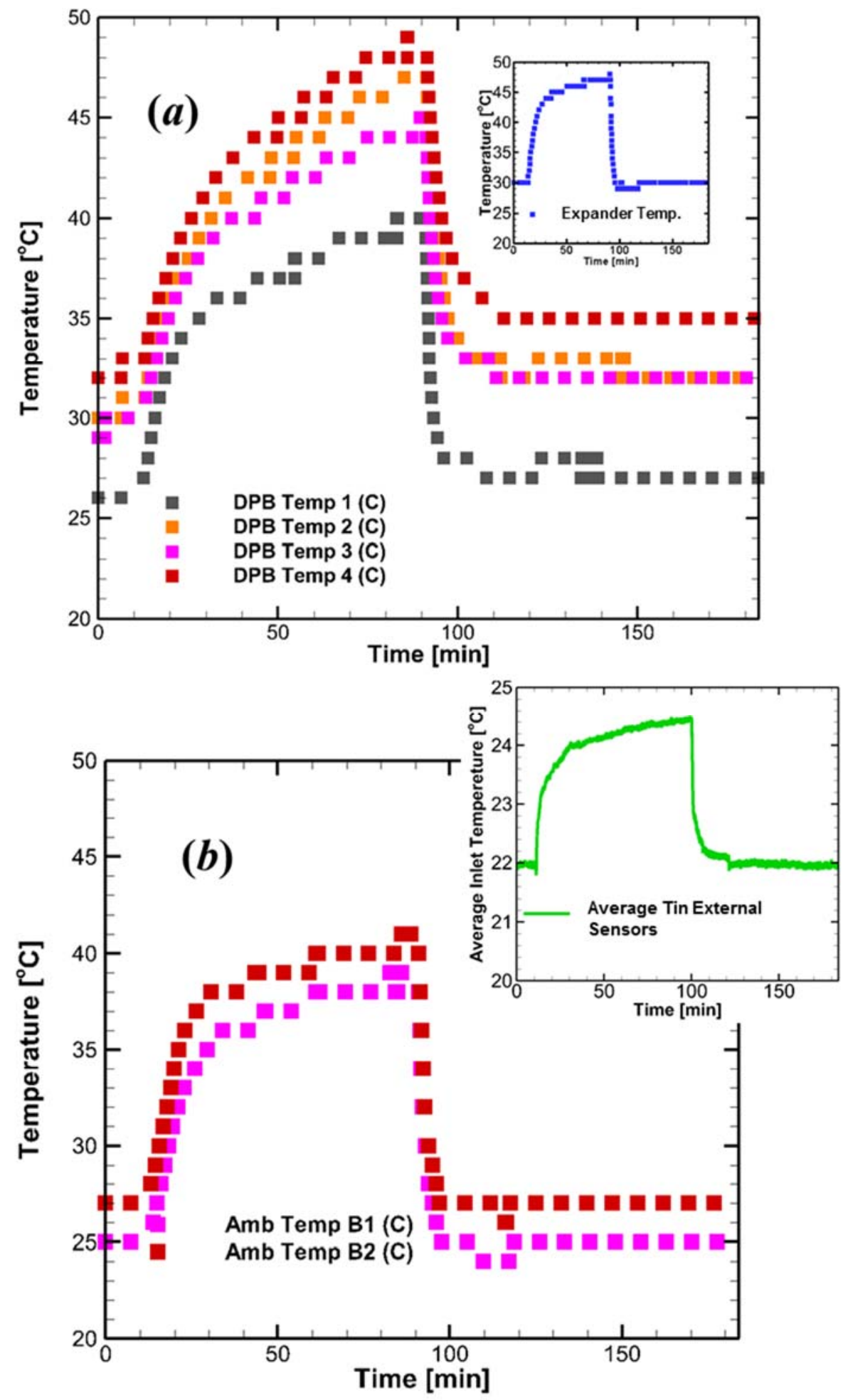

Fig. 6 (a) Drive Plane Board (DPB) and SAS Expander Board (SEB) IPMI temperature (b) IPMI ambient and external discrete sensors inlet temperature. 

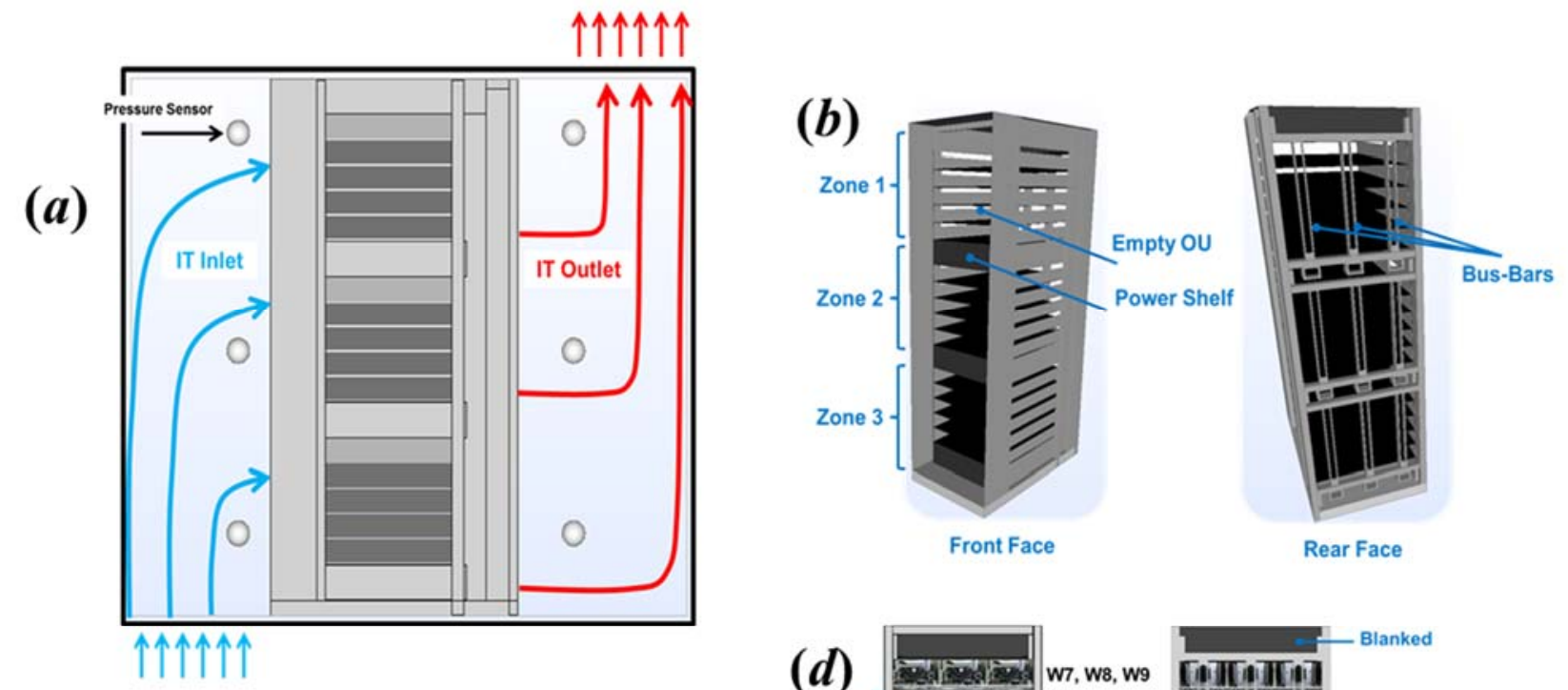

(c)
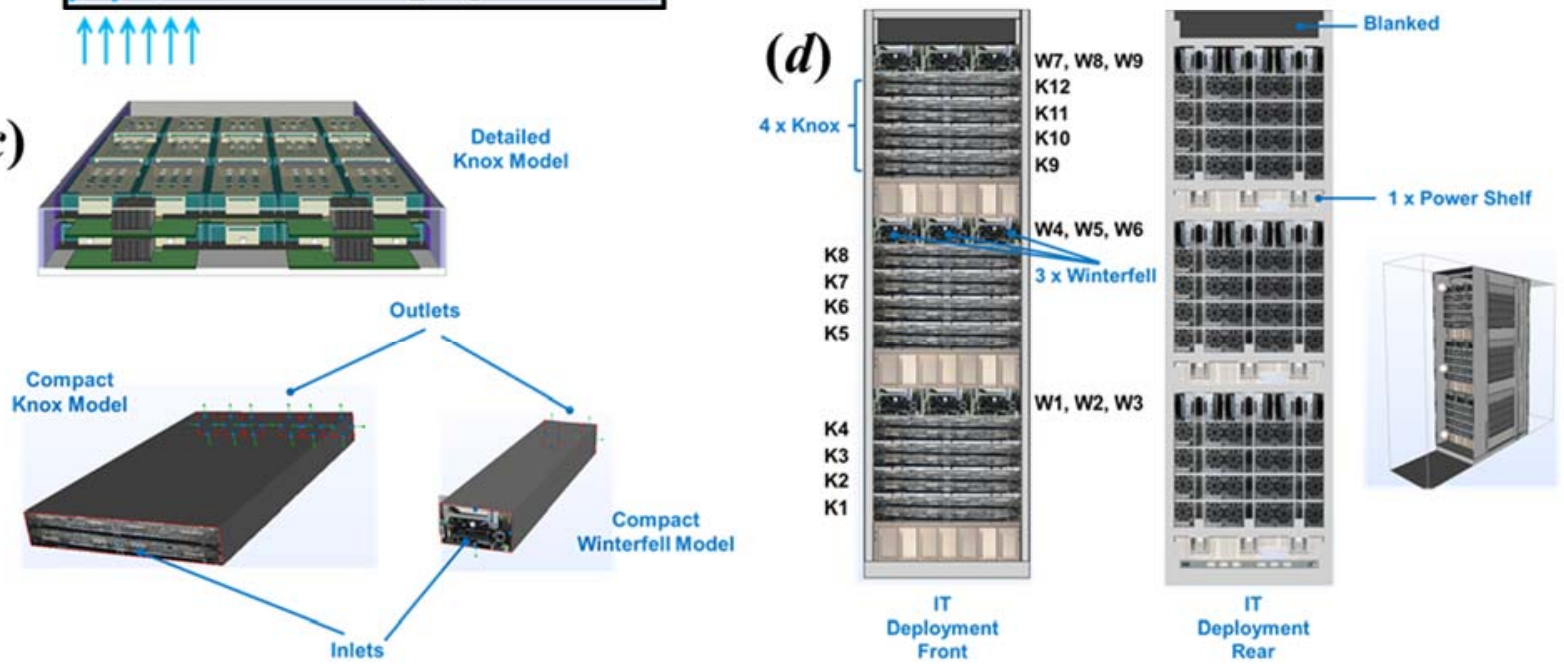

Fig. 7 (a) Rack level test chamber -side view- (b) open rack (OR) model (c) detailed and compact models (d) storage/server IT deployment 

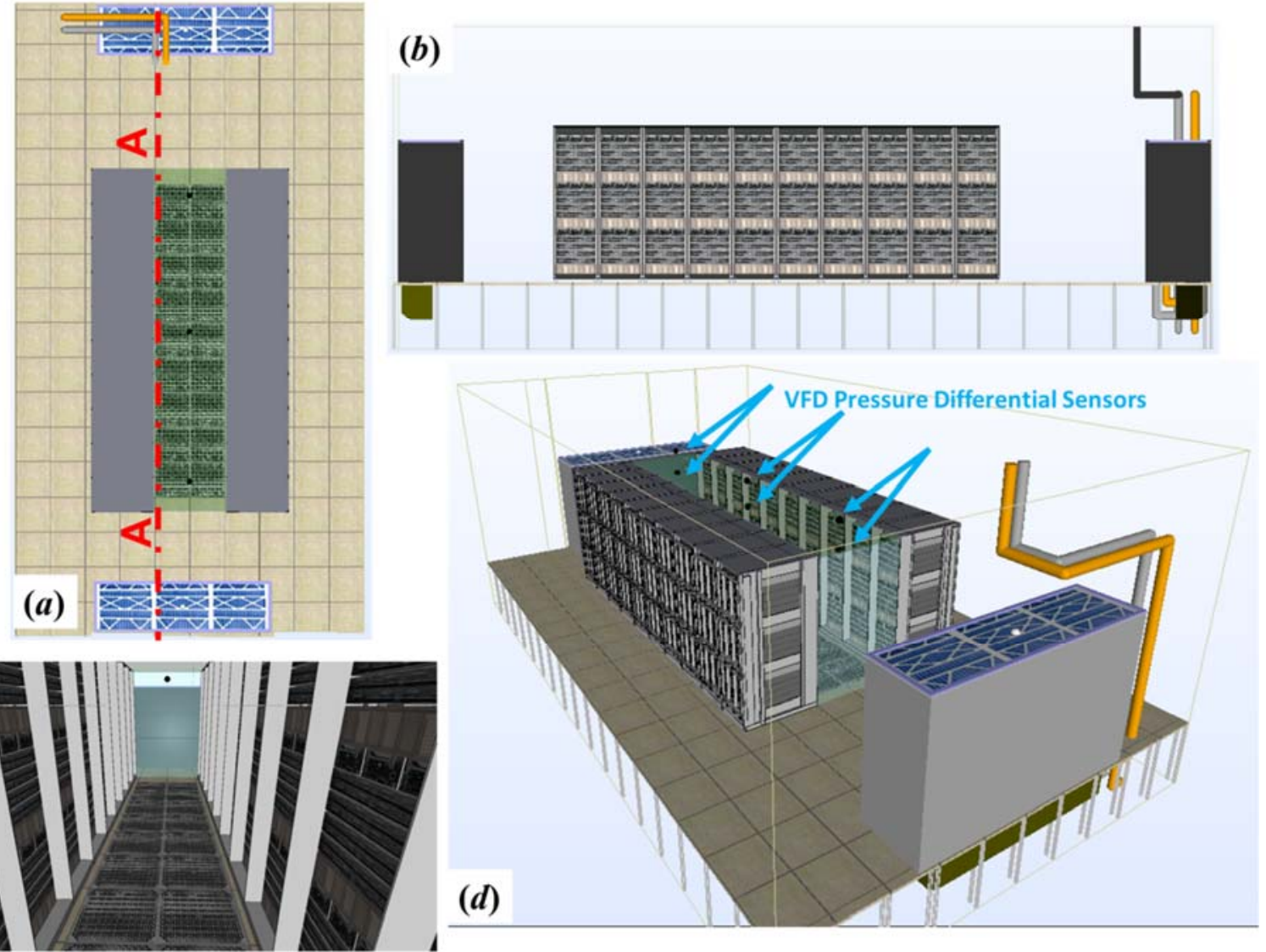

Fig. 8 (a) Top view of storage POD (b) cross section A-A view (c) deployment inside CAC (d) isometric view and pressure sensor locations. 

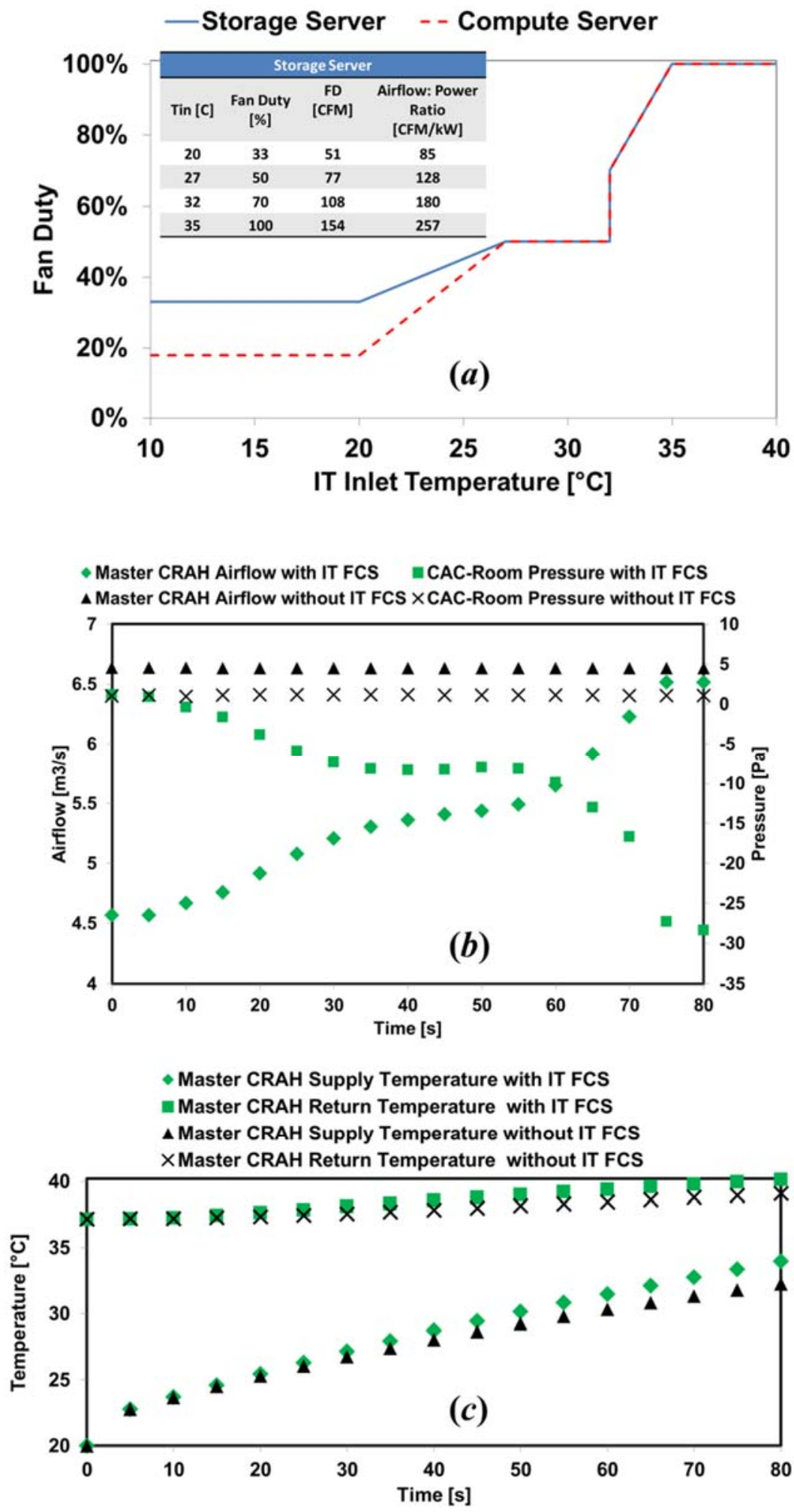

Fig. 9 (a) Generic function for IT FCS (b) pressure differential and CRAH airflow during chiller failure (b) master CRAH supply and return temperature variation. 
Without IT FCS Model

ASHRAE 2011 Class A1 Temp. (C)

T $T>32$

$27<\mathrm{T}<32$

$18<T<27$

$15<\mathrm{T}<18$

$t=0 \mathrm{~s}$$$
\mathbf{t}=\mathbf{0}
$$

ASHRAE 2011 Class A1 Temp. (C)

$T>32$

$27<\mathrm{T}<32$

$18<T<27$

$15<\mathrm{T}<18$

$\mathrm{T}<15$

$t=25 \mathrm{~s}$

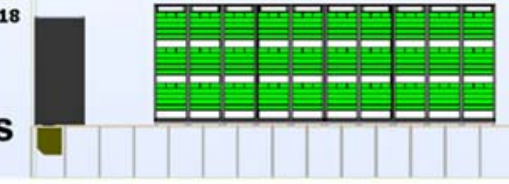

ASHRAE 2011 Class A1 Temp. (C)

$T>32$

$27<\mathrm{T}<32$

$18<T<27$

$15<T<18$
$T<15$

$\mathrm{t}=40 \mathrm{~s}$

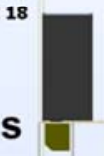

(a)

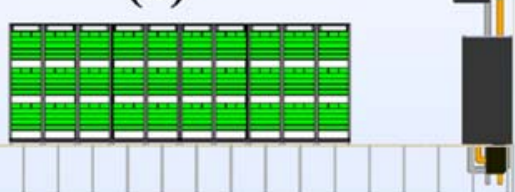

ASHRAE 2011 Class A1 Temp. (C)

$\mathrm{T}>32$

$27<\mathrm{T}<32$

$18<T<27$

$15<\mathrm{T}<18$

$T<15$

$\mathrm{t}=\mathbf{5 0} \mathrm{s}$
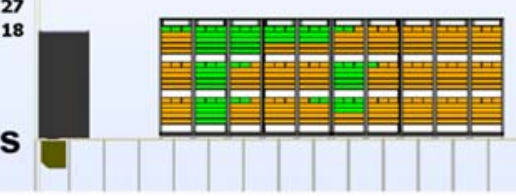

ASHRAE 2011 Class A1 Temp. (C)

$\mathbf{T}>\mathbf{3 2}$

$27<\mathrm{T}<32$

$18<\mathrm{T}<27$

$15<T<18$

$T<15$

$t=75 \mathrm{~s}$
With IT FCS Model

ASHRAE 2011 Class A1 Temp. (C)

$\mathrm{T}>32$

$27<\mathrm{T}<32$

$18<T<27$

$15<T<18$

$T<15$

(b)

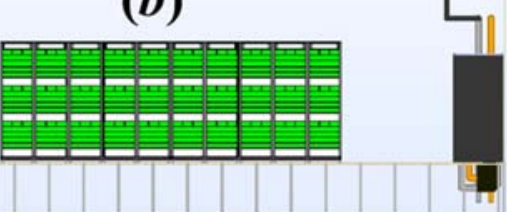

ASHRAE 2011 Class A1 Temp. (C)

$\mathrm{T}>32$

$27<$ T $<32$

$18<T<27$

$15<T<18$

$T<15$
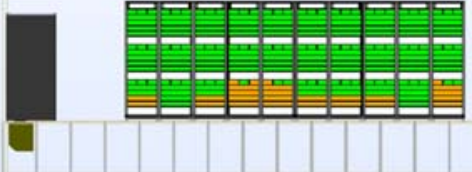

ASHRAE 2011 Class A1 Temp. (C)

$T>32$

$27<\mathrm{T}<32$

$18<T<27$

$15<T<18$
$T<15$
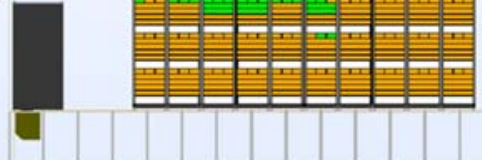

ASHRAE 2011 Class A1 Temp. (C)

$T>32$

$27<T<32$

$18<T<27$

$15<T<18$

$\mathrm{T}<15$

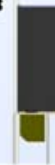

ASHRAE 2011 Class A1 Temp. (C)

$T>32$

$27<T<32$

$18<T<27$

$15<\mathrm{T}<18$
$T<15$

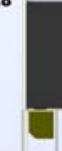

Fig. 10 IT inlet temperatures based on ASHRAE A1 compliance at A-A during chiller failure (a) without (b) with IT fan control system. 